EESTI NSV TEADUSTE AKADEEMIA TOIMETISED. XV KOIDE

FUOSIKA-MATEMAATIKA. JA TEHNIKATEADUSTE SEERIA. 1966. NR. 4

ИЗВЕСТИЯ АҚАДЕМИИ НАУК ЭСТОНСКОН ССР. ТОМ ХV

СЕРИЯ ФИЗИКО-МАТЕМАТИЧЕСКИХ И ТЕХНИЧЕСКИХ НАУК. 1966, № 4

II. $К А P Д$

\title{
О НЕРАВЕНСТВАХ, СВЯЗЫВАЮЩИХ ЭНЕРГЕТИЧЕСКИЕ КОЭФФИЦИЕНТЫ ОПТИЧЕСКИХ ПЛЕНОК
}

В статьях А. Қауни [1,2] выведены интересные соотношения между величинами, характеризующими многослойную оптическую пленку. Это, во-первых, формула

$$
\cos \gamma_{L}=\frac{A A^{\prime}-\left(R+R^{\prime}\right) D}{2 D \sqrt{R R^{\prime}}}
$$

и, во-вторых, неравенство

$$
D \leqslant\left(1+\sqrt{R^{\prime}}\right)(1-\sqrt{R})
$$

(здесь и в дальнейшем наши обозначения отчасти отличаются от обозначений А. Кауни). $R, D$ и $A$ означают энергетические коэффициенты отражения, пропускания и поглощения пленки, а штрих означает падение света на пленку с обратной стороны. Вели. чина $\gamma_{L}$ есть экстремальное значение $\gamma$ и

$$
\gamma=\mu+\mu^{\prime}
$$

а $\mu$ есть фаза величины $\frac{r}{d}$, где $p$ и $d$-амплитудные коэффициенты отражения и пропускания, Т. е.

$$
\left.\begin{array}{l}
R=r r^{*} \\
D=d d^{*}
\end{array}\right\}
$$

и

$$
\frac{r}{d}=\sqrt{\frac{R}{D}} e^{i \mu}
$$

При выводе соотношений (1) и. (2) А. Кауни использовал условие сохранения энергии в виде

$$
R+D \leqslant 1
$$

Однако такие же или сходные соотношения можно получить и независимо от условия (6), так что они приобретают более широкий смысл. Отказываясь от условия (6), мы имеем в виду случай отрицательного поглощения в пленке. Таким образом, закон сохранения энергии, естественно, остается в силе, но не в форме (6), а в более общей форме [см. ниже, формулы (11)].

Целью настоящей статьи является вывод сходных с (1) и (2) обобщенных формул, а также некоторых новых аналогичных соотношений. В этом выводе нами будут использованы, кроме указанных выше, еще следующие обозначения: 


$$
\left.\begin{array}{c}
a \equiv \frac{r}{d}=\sqrt{\frac{R}{D}} e^{i \mu} \\
b \equiv \frac{1}{d}=\frac{1}{\sqrt{D}} e^{i \nu}
\end{array}\right\}
$$

где тильда означает пленку, сопряженную к данной, т, е. получающуюся из данной путем замены всех показателей преломленія на их комплексно-сопряженные значения.

Мы воспользуемся также следующимй основными формулами теории пленок (CM. [3]) :

$$
\left.\begin{array}{l}
b \tilde{b}^{*}-a \tilde{a}^{*}=1 \\
a^{\prime}=-\tilde{a}^{*} \\
b^{\prime}=b
\end{array}\right\}
$$

Эти формулы верны без ограничений для любой пленки, независимо от того, имеет ли она положительное, отрицательное или равное нулю ноглощение: Закон сохранения энергии пишется в виде

$$
\left.\begin{array}{l}
R+D+A=1 \\
\tilde{R}+\tilde{D}+\tilde{A}=1
\end{array}\right\}
$$

где, конечно, $R \geqslant 0, \tilde{R} \geqslant 0, D \geqslant 0, \tilde{D} \geqslant 0$, но $A$ и $\tilde{A}$ могут иметь и отрицательные значения.

\section{Вывод формул А. Кауни}

Примем за исходный пункт формулу

$$
\cos (\mu-\tilde{\mu})=\frac{a \tilde{a}^{*}+a^{*} \bar{a}}{2 \sqrt{a a^{*} \tilde{a} a^{*}}}
$$

вытекающую из (7). Преобразуем ее. Из (12), с учетом (8) и (9), следует

$$
\begin{aligned}
& 2 \sqrt{a a^{*} \tilde{a} \tilde{a}^{*}} \cos (\mu-\tilde{\mu})= \\
& =2 b b^{*}(\operatorname{ch} \varphi-1)-b b^{*}\left(b b^{*}-a a^{*}+\tilde{b} \tilde{b}^{*}-\tilde{a} \tilde{a}^{*}-2\right)+ \\
& +a \tilde{a} *\left(b * \tilde{b}-a^{*} \tilde{a}\right)+a * \tilde{a}\left(b \tilde{b}^{*}-a \tilde{a} *\right)
\end{aligned}
$$

Раскрывая скобки и перегруппировывая члены, находим

$$
2 \sqrt{a a^{*} \tilde{a} \tilde{a} *} \cos (\mu-\tilde{\mu})=2 b b^{*}(\operatorname{ch} \varphi-1)-b^{2} b^{* 2}+2 b b^{*}-
$$

$-\left(b \tilde{b}^{*}-a \tilde{a}^{*}\right)\left(b^{*} \tilde{b}-a^{*} \tilde{a}\right)-a a^{*} \tilde{a} \tilde{a}^{*}+\left(b b^{*}-1\right)\left(a a^{*}+\tilde{a} \tilde{a}^{*}\right)+a a^{*}+\tilde{a} \tilde{a}^{*}=$ 
$=2 b b^{*}(\operatorname{ch} \varphi-1)+a a^{*}+\tilde{a} \tilde{a}^{*}-\left(b b^{*}-a a^{*}-1\right)\left(b b^{*}-\tilde{a} \tilde{a}^{*}-1\right)=$ $=2 b b^{*}(\operatorname{ch} \varphi-1)+a a^{*}+a^{\prime} a^{\prime *}-\left(b b^{*}-a a^{*}-1\right)\left(b^{\prime} b^{\prime *}-a^{\prime} a^{\prime *}-1\right)=$ $=\frac{2(\operatorname{ch} \varphi-1)}{D}+\frac{R}{D}+\frac{R^{\prime}}{D^{\prime}}-\frac{A A^{\prime}}{D D^{\prime}}$.

Наконец, учитывая, что, согласно (10), $D^{\prime}=D$ и $\mu-\tilde{\mu}=\pi+\mu+\mu^{\prime}$, находим

$$
\cos \left(\mu+\mu^{\prime}\right)=\frac{\frac{A A^{\prime}}{D}-\left(R+R^{\prime}\right)}{2 \sqrt{R R^{\prime}}}-\frac{c h \varphi-1}{\sqrt{R R^{\prime}}}
$$

По поводу полученной формулы следует, прежде всего, заметить, что она теряет смысл, если $R=0$ и/или $R^{\prime}=0$. Вместо нее будем тогда иметь (полагая, без ограничения общности, $R^{\prime}=0$ )

$$
\frac{A A^{\prime}}{D}-R-2(\operatorname{ch} \varphi-1)=0
$$

или, заменяя $A^{\prime}=1-D$,

$$
\frac{A}{D}+D=2 \operatorname{ch} \varphi-1
$$

Эту формулу можно рассматривать как предельный случай формулы (13). Ее можно получить и непосредственно из формул (8) и (9). В самом деле, так как $R^{\prime}=0$ означает, что $\tilde{a}=0$, то $b \dot{b}^{*}=1$, откуда $D \tilde{D}=1$; кроме того,

$$
2 \operatorname{ch} \varphi-1=b b^{*}-a a^{*}+\tilde{b} \tilde{b}^{*}-1
$$

Так как здесь $b b^{*}-a a^{*}-1=\frac{A}{\bar{D}}$ и $\tilde{b} \tilde{b}^{*} \Rightarrow \frac{1}{\tilde{D}}=D$, то отсюда и нолучается формула (14).

Вернемся к формуле (13). Величина $ф$, согласно формуле (8), является вещественной и сh $\varphi \geqslant 1$ тогда и то:Іько тогда, если

$$
\frac{A}{D}+\frac{\tilde{A}}{\tilde{D}} \geqslant 0
$$

Можно показать (см. $\left.{ }^{4}\right]$ ), что это неравенство действительно выполняется для любой пленки, все слои которой имеют поглощение одного знака, т. е. либо только положительное, либо только отрицательное. Ниже будем везде предполагать, что это условие выполнено. Тогда из формулы (13) непосредственно вытекает формула А. Кауни (1). Однако, теперь она выведена не только для случая положительного поглощения, но и для случая отрицательного поглощения, когда неравенство (6) не выполняется. В предельном случае отсутствия поглошения, как хорошо известно, $R^{\prime}=R, A=A^{\prime}=0$ и $\varphi=0$. Следовательно, тогда, по формуле $(13), \cos \left(\mu+\mu^{\prime}\right)=-1$, что также хорошо известно.

Чтобы получить в обобщенном виде вторую формулу А. Кауни, перепишем (13) в виде следуюшего неравенства:

$$
\frac{R+R^{\prime}-\frac{A A^{\prime}}{D}}{2 \sqrt{R R^{\prime}}} \leqslant 1
$$


Подставляя сюда $A=1-R-D$ и $A^{\prime}=1-R^{\prime}-D$, находим

$$
D^{2}-2\left(1-\sqrt{R R^{\prime}}\right) D+(1-R)\left(1-R^{\prime}\right) \geqslant 0
$$

или

$$
\left[D-(1+\sqrt{R})\left(1-\sqrt{R^{\prime}}\right)\right]\left[D-(1-\sqrt{R})\left(1+\sqrt{R^{\prime}}\right)\right] \geqslant 0 .
$$

Это неравенство верно не только если $R=0$ и $R^{\prime} \neq 0$, но и при $R=0$ и/или $R^{\prime}=0$, несмотря на то, что оно получено из формулы (13), которая в этих случаях неприменима. В самом деле, если, например, $R^{\prime}=0$. то, согласно (14); имеем

$$
\frac{A}{D}+D-1 \geq 0
$$

Подставляя $A=1-R-D$, находим

$$
D^{2}-2 D+1-R \geqslant 0
$$

откуда

$$
[D-(1+\sqrt{R})][D-(1-\sqrt{R})] \geqslant 0 .
$$

Но это неравенство совпадает с неравенством (17), если положить в нем $R^{\prime}=0$. Очевидно, оно останется верным и при $R=0$, а также при $R=$ $=R^{\prime}=0$.

Не ограничивая общности, положим теперь, что

$$
R^{\prime} \leqslant R \text {. }
$$

Тогда из (17) вытекает, что должно выполняться одно из двух неравенств:

или

$$
\begin{aligned}
& D \geqslant(1+\sqrt{R})\left(1-\sqrt{R^{\prime}}\right) \\
& D \leqslant(1-\sqrt{R})\left(1+\sqrt{R^{\prime}}\right),
\end{aligned}
$$

причем, так как

$$
(1+\sqrt{R})\left(1-\sqrt{R^{\prime}}\right) \geqslant(1-\sqrt{R})\left(1+\sqrt{R^{\prime}}\right),
$$

оба они сразу выполняться не могут (кроме, конечно, тривиального предельного случая, когда везде стоит знак равенства).

Дальше нужно различать два случая.

1. Если поглошение во всех слоях положительно, то $A>0, A^{\prime}>0$. Отсюда вытекает, что неравенство (19) невозможно, так как в противнсм случае имело бы место также неравенство

$$
D \geqslant\left(1+\sqrt{R^{\prime}}\right)(1-\sqrt{R})
$$

перемножая оба, мы имели бы

$$
D^{2} \geqslant(1-R)\left(1-R^{\prime}\right) \text {, }
$$

T. e.

$$
D^{2} \geqslant(A+D)\left(A^{\prime}+D\right)
$$


Но это противоречит условию положительности поглощения. Следовательно, в этом случае

$$
\left.\begin{array}{l}
D \leqslant(1-\sqrt{R})\left(1+\sqrt{R^{\prime}}\right) \\
D \leqslant(1+\sqrt{R})\left(1-\sqrt{R^{\prime}}\right) .
\end{array}\right\}
$$

Это и есть неравенство А. Кауни (2).

2. Если поглощение во всех слоях отрицательно, то $A<0, A^{\prime}<0$. Тсгда невозможно неравенство (20), что в случае $R>1$ очевидно; если же $R<1$, то, умножая (20) на вытекающее из него неравенство

$$
D \leqslant(1+\sqrt{R})\left(1-\sqrt{R^{\prime}}\right),
$$

мы получили бы, что

T. e.

$$
\begin{aligned}
& D^{2} \leqslant(1-R)\left(1-R^{\prime}\right), \\
& D^{2} \leqslant(A+D)\left(A^{\prime}+D\right),
\end{aligned}
$$

что при $A<0, A^{\prime}<0$, так как $A+D=1-R>0$ и $A^{\prime}+D>0$, невоз. мсжно. Следовательно, в этом случае

$$
\begin{aligned}
& D \geqslant(1-\sqrt{R})\left(1+\sqrt{R^{\prime}}\right) \\
& D \geqslant(1+\sqrt{R})\left(1-\sqrt{R^{\prime}}\right) .
\end{aligned}
$$

Объединяя оба результата, запишем окончательный результат в виде обобщенного неравенства А. Кауни:

если $A \gtreqless 0$.

$$
\left.\begin{array}{l}
D \leqq(1-v \bar{R})\left(1+\dot{V} \overline{R^{\prime}}\right) \cdots \\
D \leqq(1+\sqrt{R})\left(1-\bar{V} \bar{R}^{\prime}\right)
\end{array}\right\}
$$

\section{Новые формулы}

Аналогично формуле (12), мы можем исходдить также из формулы

$$
\cos (v-\bar{v})=\frac{b \tilde{b}^{*}+b^{*} \tilde{b}}{2 \sqrt{b b^{*} \tilde{b} \tilde{b}^{*}}}
$$

Преобразуем ее аналогичным образом:

$2 \sqrt{b b^{*} \tilde{b} \tilde{b}^{*}} \cos (v-\tilde{v})=2 a a^{*}(\operatorname{ch} \varphi-1)-a a^{*}\left(b b^{*}-a a^{*}+\tilde{b} \tilde{b}^{*}-\tilde{a} \tilde{a}^{*}-2\right)+$ $+b \tilde{b}^{*}\left(\tilde{b} b^{*}-\tilde{a} a^{*}\right)+b^{*} \tilde{b}\left(b \tilde{b}^{*}-a \tilde{a}^{*}\right)=2 a a^{*}(\operatorname{ch} \varphi-1)+a^{2} a^{* 2}+2 a a^{*}+$ $+\left(b \tilde{b}^{*}-a \tilde{a}^{*}\right)\left(b^{*} \tilde{b}-a^{*} \tilde{a}\right)+b b^{*} \tilde{b} \tilde{b}^{*}-\left(a a^{*}+1\right)\left(b b^{*}+\tilde{b} \tilde{b}^{*}\right)+b b^{*}+\tilde{b} \tilde{b}^{*}=$ $=2 a a^{*}(\operatorname{ch} \varphi-1)+b b^{*}+\tilde{b} \tilde{b}^{*}+\left(b b^{*}-a a^{*}-1\right)\left(\tilde{b} \tilde{b}^{*}-a a^{*}-1\right)=$

$$
=\frac{2 R(\operatorname{ch} \varphi-1)}{D}+\frac{1}{D}+\frac{1}{\tilde{D}}+\frac{A \tilde{A}^{\prime}}{D \tilde{D}} \text {. }
$$


Ктак,

$$
\cos (v-\tilde{v})=R \sqrt{\frac{\tilde{D}}{D}}(\operatorname{ch} \varphi-1)+\frac{D+\tilde{D}+A \tilde{A}^{\prime}}{2 \sqrt{D \tilde{D}}}
$$

Из полученной- формулы вытекает, во-первых, что

$$
\cos (v-\tilde{v}) \geqslant \frac{D+\tilde{D}+A \tilde{A}^{\prime}}{2 \sqrt{D \tilde{D}}}
$$

Это неравенство аналогично первой формуле А. Кауни.

Во-вторых, имеем

$$
\frac{D+\tilde{D}+A \tilde{A}^{\prime}}{2 \sqrt{D \tilde{D}}} \leqslant 1
$$

Подставляя сюда

$$
A=1-R-D \quad \tilde{A}^{\prime}=1-\tilde{R}^{\prime}-\tilde{D},
$$

находим (учитывая, что $\frac{\tilde{R}^{\prime}}{\tilde{D}}=\frac{R}{D}$ )

$$
\left(\frac{R}{D}\right)^{2}-\left(\frac{1}{D}+\frac{1}{\tilde{D}}-2\right) \frac{R}{D}+\left(1-\frac{1}{\sqrt{D \tilde{D}}}\right)^{2} \leqslant 0
$$

Это неравенство можно переписать в виде

$$
\left[\frac{R}{D}+1-\frac{1}{2}\left(\frac{1}{D}+\frac{1}{\tilde{D}}\right)\right]^{2}+\left(\frac{1}{1 \bar{D}}-\frac{1}{\sqrt{\tilde{D}}}\right)^{2}\left[1-\frac{1}{4}\left(\frac{1}{\sqrt{\bar{D}}}+\frac{1}{\sqrt{\tilde{D}}}\right)^{2}\right] \leqslant 0
$$

Отсюда вытекает, что

$$
\frac{1}{2}\left(\frac{1}{\sqrt{D}}+\frac{1}{\sqrt{\tilde{D}}}\right) \geqslant 1
$$

Следовательно, можно положить

$$
\frac{1}{2}\left(\frac{1}{\sqrt{D}}+\frac{1}{\sqrt{\tilde{D}}}\right)=\operatorname{ch} \chi
$$

где $\chi$ вецественно. Тогда (29) принимает вид

$$
\left(\frac{R}{D}-\operatorname{ch} 2 \chi+\frac{1}{\sqrt{D \tilde{D}}}\right)^{2}-4 \operatorname{sh}^{2} \chi\left(\operatorname{ch}^{2} \chi-\frac{1}{\sqrt{D \tilde{D}}}\right) \leqslant 0
$$

откуда следует

$$
\left|\operatorname{sh} \chi-\sqrt{\operatorname{ch}^{2} \chi-\frac{1}{\sqrt{D \tilde{D}}}}\right| \leqslant \sqrt{\frac{R}{D}} \leqslant \operatorname{sh} \chi+\sqrt{\operatorname{ch}^{2} \chi-\frac{1}{\sqrt{D \tilde{D}}}} .
$$

Итак, мы получили два новых неравенства - (30) и (33). Из них можно вывести несколько дальнейших заключений. 
Во-первых, из неравенства (30) следует, что если $D \leqslant 0,25$, то $\tilde{D}$ не ограничено; если же $D>0,25$, то

$$
\tilde{D} \leqslant \frac{D}{(2 \sqrt{D}-1)^{2}}
$$

Наоборот, если $\tilde{D} \leqslant 0,25$, то $D$ не ограничено, а если $\tilde{D}>0,25$, то

$$
D \leqslant \frac{\tilde{D}}{(2 \sqrt{\tilde{D}}-1)^{2}}
$$

Во-вторых, если $D=\tilde{D}$, то $\operatorname{ch} \chi=\frac{1}{\sqrt{\bar{D}}} ;$ следовательно, $\operatorname{ch}^{2} \chi-\frac{1}{\sqrt{D \tilde{D}}}=0$ и, согласно неравенству (33),

$$
\frac{R}{D}=\operatorname{sh}^{2} \chi=\frac{1-D}{D},
$$

откуда следует, что $A=0$. Итак, равенство $D=\tilde{D}$ служит признаком отсутствия поглощения.

В-третьих, если $D \tilde{D}=1$, то сh $\chi=\frac{1+D}{2 \sqrt{D}}$ и sh $\chi=\frac{1-D}{2 \sqrt{D}}$. Тогда неравенство (33) принимаєт вид

$$
R \leqslant(1-D)^{2}
$$

\section{ЛИТЕРА T У P A}

1. Cown i e Allan R., J. Opt. Soc. America, 47, 132 (1957)

2. Cown i e Allan R., J. Opt. Soc. America, 53, 425 (1963).

3. К а р д П. Г., Оптика и спектроскопия, 9, 95 (1960).

4. К а р д П. Г., Анализ и синтез многослойных интерференционных пленок (в печати).

Тартуский государственный университет
Поступила в редакцию $11 / \mathrm{X} 1965$

\section{P. KARD}

\section{OPTILISTE KELMETE ENERGEETILISI KOEFITSIENTE SEOSTAVATEST VORRATUSTEST}

Mitmekihiliste optiliste ke'mete tecoria pōhivalemite lihtsate teisenduste teel on tuletatud võrratused (23), (30) ja (33), kus $R, D, A$ on kelme peegeldumise, läbilaskvuse ja neeldumise koefitsiendid; $R^{\prime}$ on peegeldumise koefitsient kelme teiselt küljelt ja $\sim$ tähen. dab konjugeeritud (vt. [3]) kelmet. Eelduseks on neeldumise samamärgilisus kelme kõigis kihtides.

\section{P. KARD}

\section{UBER DIE UNGLEICHUNGEN, DIE DIE ENERGETISCHEN KOEFFIZIENTEN EINES OPTISCHEN MEHRSCHICHTSYSTEMS MITEINANDER VERBINDEN}

Drei Ungleichungen, (23), (30) und (33), werden mittels einfacher Umformungen der Grundformeln der Theorie der optischen Mehrschichtsysteme abgeleitet. $R$ und $R^{\prime}$ bedeuten dort die Reflexionskoeffizienten an beiden Seiten des Schichtsystems, $D$ ist der Durchiassgrad und $A$ der Absorptionskoeffizient; bedeutet dús konjug:erte Schichtsystem (s. $\left.\left[^{3}\right]\right)$. Vorausgesetzt wird die Gleichineit des Vorzeichens der Absorption in allen Schichten des Systems. 